\title{
Sous-normalité jointe non bornée et applications
}

\author{
par
}

\author{
Olivier Demanze (Lille)
}

\begin{abstract}
T. Trent gave a new characterization of subnormality for an operator on a Hilbert space. T. Bînzar and D. Păunescu generalized this condition to commuting triples of operators. Here, we give an $n$-variable unbounded version of the above results. Theorems of this kind have also been obtained by Z. J. Jabłoński and J. Stochel.
\end{abstract}

1. Introduction. Le premier à s'intéresser au problème de la sousnormalité et à en donner une condition nécessaire et suffisante est P. R. Halmos (voir [8]). Sa condition est ensuite améliorée par J. Bram (voir [2]) : un opérateur $S$ sur un espace de Hilbert $\mathcal{H}$ est sous-normal si et seulement si il vérifie la condition de positivité suivante (appelée dans la suite condition de Halmos-Bram) :

$$
\sum_{i, j=0}^{p}\left\langle S^{i} x_{j}, S^{j} x_{i}\right\rangle \geq 0
$$

pour toute famille finie $\left(x_{0}, \ldots, x_{p}\right)$ de vecteurs de $\mathcal{H}$. Une version multiopératorielle est donnée par T. Itô (voir [9]). On peut même obtenir des puissances identiques dans les produits scalaires (voir les articles de M. Embry [6] et A. Lubin [11] pour le cas uni-dimensionnel et multi-dimensionnel respectivement).

Le cas non borné est beaucoup plus délicat à traiter. On commence par rappeler la définition d'un opérateur sous-normal dans le cas non borné. Si on note par $\mathcal{D}(S)$ le domaine de l'opérateur $S$, on dit que $S$ est sous-normal s'il existe un espace de Hilbert $\mathcal{K}$ contenant $\mathcal{H}$ et un opérateur normal $N$ dans $\mathcal{K}$ vérifiant

$$
\mathcal{D}(S) \subset \mathcal{D}(N), \quad S x=N x, \quad \forall x \in \mathcal{D}(S) .
$$

En fait, même si le domaine de définition est invariant, la condition de Halmos-Bram n'implique pas forcément la sous-normalité. Un exemple lié au problème multi-dimensionnel des moments est donné par J. Stochel et

2000 Mathematics Subject Classification: 47B20, 47A57, 47B15.

Key words and phrases: unbounded subnormal multi-operator. 
F. H. Szafraniec (voir [14]). Ils donnent aussi des conditions de sous-normalité pour des opérateurs non bornés (voir [14]-[16]).

On rappelle également la définition de la sous-normalité jointe dans ce cadre : on dit qu'une famille $\left(S_{1}, \ldots, S_{m}\right)$ est sous-normale s'il existe une famille d'opérateurs normaux $\left(N_{1}, \ldots, N_{m}\right)$ qui la prolonge et telle que ces opérateurs normaux ont les mesures spectrales qui commutent. Il existe différentes caractérisations de la sous-normalité jointe basées sur l'existence de formes sesquilinéaires vérifiant certaines conditions de positivité (voir par exemple [20], [21], [4] et surtout [12] pour les idées initiales). Le but de ce travail est d'étendre, au cas non borné et $n$-opératoriel, les résultats de T. Bînzar et D. Păunescu [1] sur les triplets d'opérateurs continus (voir également [7] pour le cas des paires d'opérateurs). Les preuves seront basées sur le calcul fonctionnel des extensions normales, plus en rapport avec les preuves initialement données par T. Trent [18] que celle de [1].

2. Sous-normalité jointe. Dans toute la suite on utilisera les notations classiques pour les multi-opérateurs et les multi-indices : si $\alpha=$ $\left(\alpha_{1}, \ldots, \alpha_{m}\right) \in \mathbb{Z}_{+}^{m}$ et si $T=\left(T_{1}, \ldots, T_{m}\right)$ est un multi-opérateur, on note par $T^{\alpha} x$ l'élément $T_{1}^{\alpha_{1}} \ldots T_{m}^{\alpha_{m}} x$. On munit $\mathbb{Z}_{+}^{m}$ de la loi produit terme à terme suivante :

$$
\alpha . \beta=\left(\alpha_{1} \beta_{1}, \ldots, \alpha_{m} \beta_{m}\right), \quad \forall(\alpha, \beta) \in \mathbb{Z}_{+}^{m} \times \mathbb{Z}_{+}^{m} .
$$

Dans toute la suite on notera par $P_{\mathcal{H}}$ la projection orthogonale sur $\mathcal{H}$.

DÉfinition 2.1. Soit $\left(S_{1}, \ldots, S_{m}\right)$ un multi-opérateur défini dans un espace de Hilbert $\mathcal{H}$. On suppose qu'il existe un sous-espace dense $\mathcal{D}$ inclus dans $\bigcap_{j} \mathcal{D}\left(S_{j}\right)$ vérifiant les conditions suivantes :

$$
S_{j}(\mathcal{D}) \subset \mathcal{D}, \quad S_{i} S_{j} x=S_{j} S_{i} x, \quad \forall x \in \mathcal{D}, i, j=1, \ldots, m .
$$

On définit alors

$$
S^{[\alpha, \beta]}=S_{1}^{* \alpha_{1}} \ldots S_{m}^{* \alpha_{m}} S_{1}^{\beta_{1}} \ldots S_{m}^{\beta_{m}}, \quad \forall(\alpha, \beta) \in \mathbb{Z}_{+}^{m} \times \mathbb{Z}_{+}^{m} .
$$

Si le multi-opérateur $S=\left(S_{1}, \ldots, S_{m}\right)$ est sous-normal (d'extension normale $N=\left(N_{1}, \ldots, N_{m}\right)$ sur un espace de Hilbert $\mathcal{K}$ ) et s'il existe $\mathcal{D}$ comme ci-dessus, on a

$$
\left\langle S^{\alpha} x, y\right\rangle=\left\langle N^{\alpha} x, y\right\rangle=\left\langle x, N^{* \alpha} y\right\rangle, \quad \forall(x, y) \in \mathcal{D} \times \mathcal{D} .
$$

Si on note $S^{\alpha *}$ l'opérateur $\left(S^{\alpha}\right)^{*}$, l'expression précédente nous permet de rappeler que $y \in \mathcal{D}\left(S^{\alpha *}\right)$ et $S^{\alpha *} y=P_{\mathcal{H}} N^{* \alpha} y$ pour tout multi-indice positif $\alpha$. En effet, par le calcul fonctionnel de $N$, on a $N^{* \alpha}=N^{\alpha *}$. On peut se référer à $[17$, Fact $\mathrm{D}]$ pour de tels résultats dans le cas d'un opérateur. Cela s'adapte à notre cadre sans difficulté majeure. Ce qui vient d'être prouvé nous permet également de vérifier que

$$
\mathcal{D} \subset \mathcal{D}\left(S^{\alpha *} S^{\beta}\right), \quad \forall(\alpha, \beta) \in \mathbb{Z}_{+}^{m} \times \mathbb{Z}_{+}^{m} .
$$


DÉFinition 2.2. Si $\left(F_{\alpha}\right)_{\alpha \in \mathbb{Z}^{m}}$ est une famille d'opérateurs bornés sur un espace de Hilbert $\mathcal{H}$ et si $S$ et $\mathcal{D}$ sont comme précédemment, on dit que cette famille est $S$-positive si pour toute famille finie $\left(a_{c, d}^{P, Q}\right)$ de nombres complexes vérifiant

$$
\sum_{c, d \geq 0} \sum_{P, Q \in \mathbb{Z}_{+}^{m}} a_{c, d}^{P, Q} \bar{\lambda}_{c} \lambda_{d} \bar{z}^{Q} z^{P} \geq 0, \quad \forall \lambda_{d} \in \mathbb{C}, \forall z \in \mathbb{C}^{m}
$$

on a la positivité suivante :

$$
\sum_{c, d \geq 0} \sum_{P, Q \in \mathbb{Z}_{+}^{m}} \sum_{K, L \in \mathbb{Z}_{+}^{m}} a_{c, d}^{P, Q}\left\langle F_{K+P-L-Q} S^{L+Q} f_{d, L}, S^{K+P} f_{c, K}\right\rangle \geq 0
$$

pour toute famille finie $\left(f_{c, K}\right)$ de $\mathcal{D}$.

Pour un multi-opérateur formé d'unitaires, on dira que la famille unitaire est $S$-positive si les puissances (positives et négatives) de ce multi-opérateur forment une famille $S$-positive.

ThÉORÈme 2.3. Soit $S=\left(S_{1}, \ldots, S_{m}\right)$ un multi-opérateur non borné défini dans un espace de Hilbert $\mathcal{H}$. Soit $\mathcal{D}$ un sous-espace vectoriel dense inclus dans $\bigcap_{j} \mathcal{D}\left(S_{j}\right)$ vérifiant les conditions suivantes:

$$
S_{j}(\mathcal{D}) \subset \mathcal{D}, \quad S_{i} S_{j} x=S_{j} S_{i} x, \quad \forall x \in \mathcal{D}, i, j=1, \ldots, m .
$$

Les assertions suivantes sont équivalentes:

(i) $\left.S\right|_{\mathcal{D}}=\left(\left.S_{1}\right|_{\mathcal{D}}, \ldots,\left.S_{m}\right|_{\mathcal{D}}\right)$ est sous-normal.

(ii) Il existe un espace de Hilbert $\mathcal{K} \supset \mathcal{H}$ et une famille commutative d'opérateurs unitaires $\left(U_{1}, \ldots, U_{m}\right)=U, S$-positive, telle que

$$
S^{\alpha *} S^{\beta} x=S^{\beta *} P_{\mathcal{H}} U^{\beta-\alpha} S^{\alpha} x, \quad \forall x \in \mathcal{D} .
$$

(iii) Il existe une famille $\left(A_{\alpha}\right)_{\alpha \in \mathbb{Z}^{m}}$ d'opérateurs, bornés sur $\mathcal{H}, S$-positive telle que

$$
S^{\alpha *} S^{\beta} x=S^{\beta *} A_{\beta-\alpha} S^{\alpha} x, \quad \forall x \in \mathcal{D} .
$$

Preuve. (i) $\Rightarrow$ (ii). On utilise le calcul fonctionnel pour les opérateurs non bornés comme dans [18]. Soit $N=\left(N_{1}, \ldots, N_{m}\right)$ une extension normale de $S$. Soit $E$ sa mesure spectrale associée. On obtient alors, pour tout $x \in \mathcal{D}$,

$$
S^{\alpha *} S^{\beta} x=P_{\mathcal{H}} N^{\alpha *} N^{\beta} x=P_{\mathcal{H}} N^{* \alpha} N^{\beta} x,
$$

d'après ce qui a été rappelé plus haut. Donc,

$$
\begin{aligned}
S^{\alpha *} S^{\beta} x & =P_{\mathcal{H}} \int \bar{z}^{\alpha} z^{\beta} d E(\bar{z}, z) x \\
& =P_{\mathcal{H}} \int z^{\alpha} \prod_{j=1}^{m} e^{-2 i \alpha_{j} \operatorname{Arg}\left(z_{j}\right)} \bar{z}^{\beta} \prod_{j=1}^{m} e^{2 i \beta_{j} \operatorname{Arg}\left(z_{j}\right)} d E(\bar{z}, z) x \\
& =P_{\mathcal{H}} N^{* \beta} \int \prod_{j=1}^{m}\left(e^{2 i \operatorname{Arg}\left(z_{j}\right)}\right)^{\beta_{j}-\alpha_{j}} d E(\bar{z}, z) S^{\alpha} x
\end{aligned}
$$


Bien évidemment, on pose pour $j=1, \ldots, m$,

$$
U_{j}=\int e^{2 i \operatorname{Arg}\left(z_{j}\right)} d E(\bar{z}, z)
$$

Ces opérateurs commutent et sont unitaires (voir par exemple [5] ou [13]). De plus, si $z \in \mathcal{D}\left(N^{* \beta}\right)=\mathcal{D}\left(N^{\beta *}\right)$, on a pour tout $x \in \mathcal{D}$,

$$
\left\langle S^{\beta} x, P_{\mathcal{H}} z\right\rangle=\left\langle N^{\beta} x, z\right\rangle=\left\langle x, N^{* \beta} z\right\rangle .
$$

On en conclut que, dans ce cas, $P_{\mathcal{H}} z \in \mathcal{D}\left(S^{\beta *}\right)$ et $S^{\beta *} P_{\mathcal{H}} z=P_{\mathcal{H}} N^{* \beta} z$. Ceci implique la relation suivante :

$$
S^{\alpha *} S^{\beta} x=S^{\beta *} P_{\mathcal{H}} U^{\beta-\alpha} S^{\alpha} x, \quad \forall x \in \mathcal{D} .
$$

Il reste à prouver que cette famille est $S$-positive. On se base également sur le calcul fonctionnel. Soit $\left(a_{c, d}^{P, Q}\right)$ une famille finie de nombres complexes vérifiant l'inégalité

$$
\sum_{c, d \geq 0} \sum_{P, Q \in \mathbb{Z}_{+}^{m}} a_{c, d}^{P, Q} \bar{\lambda}_{c} \lambda_{d} \bar{z}^{Q} z^{P} \geq 0, \quad \lambda_{d} \in \mathbb{C}, z \in \mathbb{C}^{m} .
$$

Alors, il faut prouver que

$$
\sum_{c, d \geq 0} \sum_{P, Q \in \mathbb{Z}_{+}^{m}} \sum_{K, L \in \mathbb{Z}_{+}^{m}} a_{c, d}^{P, Q}\left\langle U^{K+P-L-Q} S^{L+Q} f_{d, L}, S^{K+P} f_{c, K}\right\rangle \geq 0 .
$$

L'expression de gauche devient

$$
\sum_{c, d \geq 0} \sum_{P, Q \in \mathbb{Z}_{+}^{m}} \sum_{K, L \in \mathbb{Z}_{+}^{m}} a_{c, d}^{P, Q}\left\langle U^{-L-Q} S^{L+Q} f_{d, L}, U^{-K-P} S^{K+P} f_{c, K}\right\rangle .
$$

Ceci peut également s'écrire

$$
\begin{gathered}
\sum_{c, d \geq 0} \sum_{P, Q \in \mathbb{Z}_{+}^{m}} \sum_{K, L \in \mathbb{Z}_{+}^{m}} a_{c, d}^{P, Q}\left\langle\int e^{2 i(L+Q) \cdot \operatorname{Arg}(z)} z^{L+Q} d E(\bar{z}, z) f_{d, L},\right. \\
\left.\int e^{2 i(K+P) \cdot \operatorname{Arg}(z)} z^{K+P} d E(\bar{z}, z) f_{c, K}\right\rangle \\
=\sum_{c, d \geq 0} \sum_{P, Q \in \mathbb{Z}_{+}^{m}} a_{c, d}^{P, Q}\left\langle\int e^{2 i Q \cdot \operatorname{Arg}(z)} z^{Q} d E(\bar{z}, z) g_{d}, \int e^{2 i P \cdot \operatorname{Arg}(z)} z^{P} d E(\bar{z}, z) g_{c}\right\rangle,
\end{gathered}
$$

où les éléments $\left(g_{d}\right)_{d}$ sont donnés par

$$
g_{d}=\sum_{L \in \mathbb{Z}_{+}^{m}} \int^{2 i L \cdot \operatorname{Arg}(z)} z^{L} d E(\bar{z}, z) f_{d, L}, \quad d \geq 0 .
$$

La combinaison des produits scalaires devient alors

$$
\sum_{c, d \geq 0}\left\langle\int \sum_{P, Q \in \mathbb{Z}_{+}^{m}} a_{c, d}^{P, Q}\left(e^{2 i \operatorname{Arg}(z)} z\right)^{Q}{\overline{\left(e^{2 i \operatorname{Arg}(z)} z\right)}}^{P} d E(\bar{z}, z) g_{d}, g_{c}\right\rangle .
$$

On peut ensuite utiliser des idées de J. Stochel et F. H. Szafraniec [15]. On ne donne que les étapes principales de la démonstration. On note $\mu$ la mesure positive suivante : $\mu(\tau)=\sum_{d}\left\langle E(\tau) g_{d}, g_{d}\right\rangle$. 
Par le théorème de Radon-Nikodym, on montre que les mesures complexes $\left(\left\langle E(*) g_{c}, g_{d}\right\rangle\right)_{c, d}$ sont absolument continues. Donc il existe une famille de fonctions boréliennes $\left(F_{c, d}\right)_{c, d}$ vérifiant

$$
\left\langle E(\tau) g_{c}, g_{d}\right\rangle=\int_{\tau} F_{c, d}(\bar{z}, z) d \mu(\bar{z}, z) .
$$

On montre que $\sum F_{c, d} \bar{\lambda}_{c} \lambda_{d} \geq 0$, c'est-à-dire que $\left(F_{c, d}\right)_{c, d}$ est de type positif. On pose

$$
P_{c, d}(\bar{z}, z)=\sum_{P, Q \in \mathbb{Z}_{+}^{m}} a_{c, d}^{P, Q}\left(e^{2 i \operatorname{Arg}(z)} z\right)^{Q}{\overline{\left(e^{2 i \operatorname{Arg}(z)} z\right)}}^{P} .
$$

Par notre hypothèse la famille $\left(P_{c, d}\right)_{c, d}$ est également de type positif. En utilisant le produit de Schur, on obtient la même propriété pour la famille $\left(F_{c, d} P_{c, d}\right)_{c, d}$. Et en appliquant ceci à la famille $\left(\lambda_{c}=1\right)$, on obtient que

$$
\sum_{c, d \geq 0} F_{c, d}(\bar{z}, z) P_{c, d}(\bar{z}, z) \geq 0
$$

ceci $\mu$-presque partout. Or, on a

$$
\begin{aligned}
\sum_{c, d \geq 0}\left\langle\int \sum_{P, Q \in \mathbb{Z}_{+}^{m}} a_{c, d}^{P, Q}\left(e^{2 i \operatorname{Arg}(z)} z\right)^{Q}{\overline{\left(e^{2 i \operatorname{Arg}(z)} z\right)}}^{P} d E(\bar{z}, z) g_{d}, g_{c}\right\rangle & \\
& =\int \sum_{c, d \geq 0} F_{c, d}(\bar{z}, z) P_{c, d}(\bar{z}, z) d \mu(\bar{z}, z) \geq 0,
\end{aligned}
$$

ce qui permet de déduire la $S$-positivité de la famille d'opérateurs unitaires.

(ii) $\Rightarrow$ (iii). On pose

$$
A_{\gamma}=\left.P_{\mathcal{H}} U_{1}^{\gamma_{1}} \ldots U_{m}^{\gamma_{m}}\right|_{\mathcal{H}}, \quad \gamma \in \mathbb{Z}^{m} .
$$

Cette famille d'opérateurs vérifie clairement les égalités suivantes :

$$
S^{\alpha *} S^{\beta} x=S^{\beta *} A_{\beta-\alpha} S^{\alpha} x, \quad \forall x \in \mathcal{D} .
$$

De plus, cette famille est $S$-positive car la famille commutative d'opérateurs unitaires $\left(U_{1}, \ldots, U_{m}\right)$ l'est.

(iii) $\Rightarrow$ (i). Pour démontrer cette dernière implication, on va utiliser une version multi-opératorielle d'un résultat de J. Stochel et F. H. Szafraniec (voir [15] et [3]). Soit une famille finie $\left(a_{c, d}^{P, Q}\right)$ de nombres complexes vérifiant

$$
\sum_{c, d \geq 0} \sum_{P, Q \in \mathbb{Z}_{+}^{m}} a_{c, d}^{P, Q} \bar{\lambda}_{c} \lambda_{d} \bar{z}^{Q} z^{P} \geq 0, \quad \lambda_{d} \in \mathbb{C}, z \in \mathbb{C}^{m} .
$$

On a la positivité suivante :

$$
\sum_{c, d \geq 0} \sum_{P, Q \in \mathbb{Z}_{+}^{m}} \sum_{K, L \in \mathbb{Z}_{+}^{m}} a_{c, d}^{P, Q}\left\langle A_{K+P-L-Q} S^{L+Q} f_{d, L}, S^{K+P} f_{c, K}\right\rangle \geq 0
$$

pour toute famille $\left(f_{c, K}\right)$ de $\mathcal{D}$. La partie gauche de cette expression devient 
(après avoir noté que le vecteur $A_{K+P-L-Q} S^{L+Q} f_{d, L}$ est dans $\mathcal{D}\left(S^{(K+P) *}\right)$ )

$$
\begin{aligned}
\sum_{c, d \geq 0} \sum_{P, Q \in \mathbb{Z}_{+}^{m}} \sum_{K, L \in \mathbb{Z}_{+}^{m}} a_{c, d}^{P, Q}\left\langle S^{(K+P) *} A_{K+P-L-Q} S^{L+Q} f_{d, L}, f_{c, K}\right\rangle \\
=\sum_{c, d \geq 0} \sum_{P, Q \in \mathbb{Z}_{+}^{m}} \sum_{K, L \in \mathbb{Z}_{+}^{m}} a_{c, d}^{P, Q}\left\langle S^{(L+Q) *} S^{K+P} f_{d, L}, f_{c, K}\right\rangle \\
=\sum_{c, d \geq 0} \sum_{P, Q \in \mathbb{Z}_{+}^{m}} \sum_{K, L \in \mathbb{Z}_{+}^{m}} a_{c, d}^{P, Q}\left\langle S^{K+P} f_{d, L}, S^{L+Q} f_{c, K}\right\rangle .
\end{aligned}
$$

Donc, par le critère précédemment cité (voir [3]), $S$ est un multi-opérateur sous-normal.

Définition 2.4. Soit $S$ un opérateur dans $\mathcal{H}$ non nécessairement borné et soit $f$ un élément de $\mathcal{D}^{\infty}(S)$. On dit que $f$ est un vecteur borné de $S$ s'il existe $a=a(f)>0$ et $c=c(f)>0$ tels que

$$
\left\|S^{n} f\right\| \leq a c^{n}, \quad \forall n \in \mathbb{Z}_{+} .
$$

On dit également que $f$ est un vecteur analytique s'il existe $t=t(f)>0$ tel que

$$
\sum_{n=1}^{\infty} \frac{\left\|S^{n} f\right\|}{n !} t^{n}<\infty .
$$

Enfin, on dira que $f$ est un vecteur quasi-analytique s'il vérifie

$$
\sum_{n=1}^{\infty}\left\|S^{n} f\right\|^{-1 / n}=+\infty .
$$

On notera respectivement par $\mathcal{B}(S), \mathcal{A}(S)$ et $\mathcal{Q}(S)$ les ensembles des vecteurs bornés, analytiques et quasi-analytiques de $S$. Ces ensembles sont tous stables par $S$. Pour les deux premiers, c'est évident. Pour $\mathcal{Q}(S)$, cela découle d'une inégalité de Carleman (voir [14]).

Le fait important, ici, est que $\mathcal{B}(S)$ et $\mathcal{A}(S)$ sont des espaces vectoriels. On rappelle maintenant un résultat de J. Stochel et F. H. Szafraniec sur les opérateurs à domaines quasi-analytiques.

ThÉorème 2.5 (Stochel-Szafraniec). Soit $S=\left(S_{1}, \ldots, S_{m}\right)$ un multiopérateur non nécessairement borné défini dans un espace de Hilbert $\mathcal{H}$. Soit $\mathcal{D}$ un sous-espace vectoriel dense inclus dans $\bigcap_{j} \mathcal{D}\left(S_{j}\right)$ vérifiant

$$
S_{j}(\mathcal{D}) \subset \mathcal{D}, \quad S_{i} S_{j} x=S_{j} S_{i} x, \quad \forall x \in \mathcal{D}, i, j=1, \ldots, m .
$$

Supposons que $S$ vérifie les conditions suivantes:

(i) $\mathcal{D}$ est engendré par l'ensemble $\left\{S^{\alpha} f: \alpha \in \mathbb{Z}_{+}^{n}, f \in \bigcap_{j} \mathcal{Q}\left(S_{j}\right)\right\}$.

(ii) $S$ vérifie la positivité de Itô, i.e.

$$
\sum\left\langle S^{\alpha} f_{\beta}, S^{\beta} f_{\alpha}\right\rangle \geq 0, \quad \forall\left(f_{\alpha}\right) \subset \mathcal{D} .
$$

Alors le multi-opérateur $\left.S\right|_{\mathcal{D}}=\left(\left.S_{1}\right|_{\mathcal{D}}, \ldots,\left.S_{m}\right|_{\mathcal{D}}\right)$ est sous-normal. 
On peut alors donner le résultat suivant :

ThÉORÈme 2.6. Soit $S=\left(S_{1}, \ldots, S_{m}\right)$ un multi-opérateur non borné défini dans un espace de Hilbert $\mathcal{H}$. Soit $\mathcal{D}$ un sous-espace vectoriel dense inclus dans $\bigcap_{j} \mathcal{D}\left(S_{j}\right)$ vérifiant

$$
S_{j}(\mathcal{D}) \subset \mathcal{D}, \quad S_{i} S_{j} x=S_{j} S_{i} x, \quad \forall x \in \mathcal{D}, i, j=1, \ldots, m .
$$

On suppose, de plus, que

$$
\mathcal{D} \subset \bigcap_{j=1}^{m} \mathcal{Q}\left(S_{j}\right) .
$$

Les assertions suivantes sont équivalentes:

(i) $\left.S\right|_{\mathcal{D}}=\left(\left.S_{1}\right|_{\mathcal{D}}, \ldots,\left.S_{m}\right|_{\mathcal{D}}\right)$ est sous-normal.

(ii) Il existe un espace de Hilbert $\mathcal{K} \supset \mathcal{H}$ et une famille commutative d'opérateurs unitaires $\left(U_{1}, \ldots, U_{m}\right)$ telle que

$$
S^{\alpha *} S^{\beta} x=S^{\beta *} P_{\mathcal{H}} U^{\beta-\alpha} S^{\alpha} x, \quad \forall x \in \mathcal{D}, \forall \alpha . \beta=0 .
$$

(iii) Il existe une famille $\left(A_{\alpha}\right)_{\alpha \in \mathbb{Z}^{m}}$ d'opérateurs, bornés sur $\mathcal{H}$, telle que

$$
\begin{aligned}
& S^{\alpha *} S^{\beta} x=S^{\beta *} A_{\beta-\alpha} S^{\alpha} x, \quad \forall x \in \mathcal{D}, \forall \alpha . \beta=0, \\
& \sum_{\alpha, \beta \geq 0}\left\langle A_{\beta-\alpha} f_{\beta}, f_{\alpha}\right\rangle \geq 0, \quad f_{\alpha} \in \mathcal{D} .
\end{aligned}
$$

Preuve. (i) $\Rightarrow$ (ii). Il suffit d'utiliser la même preuve que pour le théorème 2.3. En ce qui concerne l'implication (ii) $\Rightarrow$ (iii), on pose $A_{\gamma}=\left.P_{\mathcal{H}} U^{\gamma}\right|_{\mathcal{H}}$.

(iii) $\Rightarrow$ (i). La méthode utilisée ici est naturelle et s'apparente à celle utilisée dans le Théorème 3.1 de [10]. Pour tout $(\alpha, \beta) \in \mathbb{Z}^{m} \times \mathbb{Z}^{m}$, on pose

$$
(\underline{\alpha, \beta})=\left(\min \left(\alpha_{1}, \beta_{1}\right), \ldots, \min \left(\alpha_{m}, \beta_{m}\right)\right) \in \mathbb{Z}^{m} .
$$

On peut alors calculer les sommes suivantes (en notant qu'il faut faire attention car la première propriété sur les opérateurs $\left(A_{\alpha}\right)_{\alpha \in \mathbb{Z}^{m}}$ n'est valable que lorsque $\alpha \cdot \beta=0)$ :

$$
\begin{aligned}
\sum_{\alpha, \beta \geq 0}\left\langle S^{\beta} f_{\alpha}, S^{\alpha} f_{\beta}\right\rangle & =\sum_{\alpha, \beta \geq 0}\left\langle S^{\beta-\underline{(\alpha, \beta)}} S^{(\underline{\alpha, \beta})} f_{\alpha}, S^{\alpha-\underline{(\alpha, \beta)}} S^{(\underline{\alpha, \beta})} f_{\beta}\right\rangle \\
& =\sum_{\alpha, \beta \geq 0}\left\langle S^{(\alpha-\underline{(\alpha, \beta)}) *} S^{\beta-(\underline{\alpha, \beta)}} S^{(\underline{\alpha, \beta)}} f_{\alpha}, S^{(\underline{\alpha, \beta})} f_{\beta}\right\rangle \\
& =\sum_{\alpha, \beta \geq 0}\left\langle S^{(\beta-(\underline{\alpha, \beta})) *} A_{\alpha-\beta} S^{\alpha-(\underline{\alpha, \beta})} S^{(\underline{\alpha, \beta})} f_{\alpha}, S^{(\underline{\alpha, \beta})} f_{\beta}\right\rangle \\
& =\sum_{\alpha, \beta \geq 0}\left\langle A_{\alpha-\beta} S^{\alpha} f_{\alpha}, S^{\beta} f_{\beta}\right\rangle=\sum_{\alpha, \beta \geq 0}\left\langle A_{\alpha-\beta} g_{\alpha}, g_{\beta}\right\rangle \geq 0
\end{aligned}
$$


avec $g_{\alpha}=S^{\alpha} f_{\alpha}$, pour tout $\alpha \in \mathbb{Z}_{+}^{m}$. Par le théorème de J. Stochel et F. H. Szafraniec, cité précédemment, on obtient que $\left.S\right|_{\mathcal{D}}=\left(\left.S_{1}\right|_{\mathcal{D}}, \ldots,\left.S_{m}\right|_{\mathcal{D}}\right)$ est sous-normal.

REMARQUes 2.7. On sait que l'ensemble des vecteurs quasi-analytiques n'est pas un espace vectoriel. Mais on peut en particulier appliquer le résultat précédent au cas où le sous-espace $\mathcal{D}$ est l'intersection des ensembles analytiques, ce qui est stable par linéarité.

On peut restreindre la condition de positivité aux cas où les $\alpha, \beta$ vérifient $\alpha . \beta=0$, car on utilise la positivité de Itô (comme dans [1]).

Comme corollaire du résultat précédent, on obtient dans le cas borné

Corollaire 2.8. Soit $S=\left(S_{1}, \ldots, S_{m}\right)$ un multi-opérateur borné commutatif défini sur un espace de Hilbert $\mathcal{H}$. Les assertions suivantes sont équivalentes :

(i) $S=\left(S_{1}, \ldots, S_{m}\right)$ est sous-normal.

(ii) Il existe un espace de Hilbert $\mathcal{K} \supset \mathcal{H}$ et une famille commutative d'opérateurs unitaires $\left(U_{1}, \ldots, U_{m}\right)$ telle que

$$
S^{[\alpha, \beta]} x=S^{* \beta} P_{\mathcal{H}} U^{\beta-\alpha} S^{\alpha} x, \quad \forall x \in \mathcal{H}, \forall \alpha \cdot \beta=0 .
$$

(iii) Il existe une famille $\left(A_{\alpha}\right)_{\alpha \in \mathbb{Z}^{m}}$ d'opérateurs, bornés sur $\mathcal{H}$, telle que

$$
\begin{aligned}
& S^{[\alpha, \beta]} x=S^{* \beta} A_{\beta-\alpha} S^{\alpha} x, \quad \forall x \in \mathcal{H}, \forall \alpha \cdot \beta=0, \\
& \sum_{\alpha, \beta \geq 0}\left\langle A_{\beta-\alpha} f_{\beta}, f_{\alpha}\right\rangle \geq 0, \quad f_{\alpha} \in \mathcal{H} .
\end{aligned}
$$

Preuve. Il suffit d'utiliser le résultat précédent en notant que, puisque nous avons des opérateurs bornés, tout élément de $\mathcal{H}$ est un vecteur borné et donc inclus dans l'ensemble des vecteurs quasi-analytiques.

En particulier, on obtient le Théorème 1 de T. Bînzar et D. Păunescu, pour $m=3$. En effet, par le passage aux adjoints (sur les relations) on peut diminuer le nombre de conditions :

$$
S^{[\beta, \alpha]}=\left(S^{[\alpha, \beta]}\right)^{*} .
$$

REmARque 2.9. On peut noter également que le Corollaire 2.8 peut être relier au Théorème 3.1 et au Corollaire 3.2 de [10]. En effet, le fait de travailler sur des sous-ensembles $\omega$ et $\tilde{\omega}$ complémentaires de $\{1, \ldots, m\}$ est traduit dans notre cadre par la condition $\alpha \cdot \beta=0$.

3. Remarque sur le problème des moments vectoriel. Ce problème classique a déjà été traité par plusieurs auteurs. Étant donné une famille de vecteurs $\left(h_{\alpha}\right)_{\alpha \in \mathbb{Z}_{+}^{m}}$ d'un espace de Hilbert $\mathcal{H}$, peut-on trouver une famille commutative d'opérateurs normaux vérifiant $h_{\alpha}=S^{\alpha} h_{0}, \forall \alpha \in \mathbb{Z}_{+}^{m}$ ? Dans toute la suite, on notera par $\left(e_{1}, \ldots, e_{m}\right)$ la base canonique de $\mathbb{C}^{m}$. 
En utilisant des méthodes similaires à celles de [1], on peut prouver le Théorème 3.1 ci-dessous. Il faut noter qu'un résultat de ce type ne demandant que des conditions sur la famille de vecteurs de $\mathcal{H}$ a été prouvé récemment dans [10] (à paraître au moment de la rédaction de ce travail). Je tiens à remercier le référé de m'avoir signalé ce résultat que je ne connaissais pas lors de la rédaction de ce travail. C'est pourquoi on ne donnera pas de démonstration du Théorème 3.1 ici.

THÉORÈme 3.1. Soit $\mathcal{H}$ un espace de Hilbert et soit $\left(h_{\alpha}\right)_{\alpha \in \mathbb{Z}_{+}^{m}}$ une famille d'éléments de $\mathcal{H}$ qui engendre l'espace de Hilbert $\mathcal{H}$ telle que l'on ait $(j=$ $1, \ldots, m)$ :

$$
\forall \alpha \in \mathbb{Z}_{+}^{m}, \exists t=t_{\alpha} \quad \sum_{n=1}^{+\infty}\left\|h_{\alpha+n e_{j}}\right\| t^{n}(n !)^{-1}<+\infty .
$$

Les assertions suivantes sont équivalentes:

(i) Il existe un opérateur sous-normal $S=\left(S_{1}, \ldots, S_{m}\right)$ non nécessairement borné vérifiant $h_{\alpha}=S^{\alpha} h_{0}, \forall \alpha \in \mathbb{Z}_{+}^{m}$.

(ii) Il existe une suite de vecteurs $\left(h_{\alpha, \beta}\right)_{\alpha, \beta \in \mathbb{Z}_{+}^{m}}$ de $\mathcal{H}$ vérifiant les propriétés suivantes:

- $h_{\alpha, 0}=h_{\alpha}, \forall \alpha \in \mathbb{Z}_{+}^{m}$,

- $\left\langle h_{\alpha, \beta}, h_{\delta}\right\rangle=\left\langle h_{\alpha}, h_{\delta+\beta}\right\rangle, \forall \alpha, \delta, \beta \in \mathbb{Z}_{+}^{m}$,

- $\left\|\sum_{\alpha, \beta \geq 0} c_{\alpha, \beta} h_{\alpha, \beta}\right\|^{2} \leq \sum_{\alpha, \beta, \alpha^{\prime}, \beta^{\prime} \geq 0} c_{\alpha, \beta} c_{\alpha^{\prime}, \beta^{\prime}}\left\langle h_{\alpha+\beta^{\prime}}, h_{\beta+\alpha^{\prime}}\right\rangle$.

En particulier, on obtenait une généralisation au cas des multi-opérateurs quelconques du résultat de [1], où le cas des triplets d'opérateurs est étudié. Des conditions explicites pour un tel résultat ont déjà été obtenues par Z. J. Jabłoński et J. Stochel (voir le Théorème 6.1 et la Proposition 6.3 de [10]).

En utilisant les méthodes du Théorème 3.1, on peut donner une version opératorielle de ce théorème. De tels types de résultats ont été obtenus par F.-H. Vasilescu (voir Théorème 3.7 de [19]). On obtient alors une généralisation d'un théorème de [1]. Pour énoncer le résultat suivant, on a besoin de quelques notations. Si $T$ est un opérateur non nécessairement borné dans $\mathcal{H}$, on note par $\mathcal{D}^{\infty}(T)$ l'intersection des domaines des itérés de $T$. De plus l'ensemble $\operatorname{Vect}(\mathcal{A})(\mathcal{A} \subset \mathcal{H})$ représente l'espace vectoriel engendré par les vecteurs de $\mathcal{A}$. Pour ce résultat également, Z. J. Jabłoński et J. Stochel ont obtenu un critère explicite plus général (voir par exemple le Théorème 5.5 ou Théorème 5.6 de [10]).

ThÉORÈme 3.2. Soit $\mathcal{H}$ un espace de Hilbert et soit $\left(T_{\alpha}\right)_{\alpha \in \mathbb{Z}_{+}^{m}}$ une famille d'opérateurs non nécessairement bornés dans $\mathcal{H}$. On suppose qu'il existe un sous-espace invariant $\mathcal{D}$ dense vérifiant $\mathcal{D} \subset \mathcal{D}^{\infty}\left(T_{\alpha}\right) \cap \mathcal{D}^{\infty}\left(T_{\alpha}^{*}\right)$ (pour tout $\alpha$ ) 
et tel que $\operatorname{Vect}\left(T_{\alpha}(\mathcal{D})\right)_{\alpha}$ soit dense dans $\mathcal{H}$. On suppose de plus que pour tout $x \in \operatorname{Vect}\left(T_{\alpha}(\mathcal{D})\right)_{\alpha}$, il existe $t_{x} \neq 0$ tel que $\sum_{n=1}^{+\infty}\left\|T_{\alpha+n e_{j}} x\right\| t_{x}^{n}(n !)^{-1}<+\infty$. Alors les assertions suivantes sont équivalentes :

(i) Il existe un opérateur sous-normal $S=\left(S_{1}, \ldots, S_{m}\right)$ non nécessairement borné vérifiant $T_{\alpha}=S^{\alpha} T_{0}, \forall \alpha \in \mathbb{Z}_{+}^{m}$.

(ii) Il existe une suite d'opérateurs $\left(T_{\alpha, \beta}\right)_{\alpha, \beta \in \mathbb{Z}_{+}^{m}}$ défini sur un domaine contenant $\mathcal{D}$ vérifiant les propriétés suivantes:

- $T_{\alpha, 0}=T_{\alpha}, \forall \alpha \in \mathbb{Z}_{+}^{m}$,

- $T_{\delta}^{*} T_{\alpha, \beta} x=T_{\delta+\beta}^{*} T_{\alpha} x, \forall x \in \mathcal{D}, \forall \alpha, \delta, \beta \in \mathbb{Z}_{+}^{m}$,

- $\left\|\sum_{\alpha, \beta \geq 0} T_{\beta, \alpha} x_{\alpha, \beta}\right\|^{2} \leq \sum_{\alpha, \beta, \alpha^{\prime}, \beta^{\prime} \geq 0}\left\langle T_{\alpha^{\prime}+\beta} x_{\alpha, \beta}, T_{\beta^{\prime}+\alpha} x_{\alpha^{\prime}, \beta^{\prime}}\right\rangle$.

En particulier, on obtenait une généralisation, au cas des multi-opérateurs quelconques, d'un résultat de T. Bînzar et D. Păunescu. Ce résultat était déjà obtenu par Z. J. Jabłoński et J. Stochel (voir la Proposition 9.1 de $[10])$.

Remerciements. L'auteur voudrait remercier le référé de lui avoir signalé un article à paraître (au moment de la rédaction de ce travail, [10]), dont il n'avait pas connaissance, dans lequel Z. J. Jabłoński et J. Stochel donnent des critères explicites et plus généraux que ceux énoncés dans la dernière section.

\section{Références}

[1] T. Bînzar and D. Păunescu, Commuting triples of subnormal operators and related moment problems, in: Recent Advances in Operator Theory, Operator Algebras, and Their Applications, Oper. Theory Adv. Appl. 153, Birkhäuser, 2005, 39-59.

[2] J. Bram, Subnormal operators, Duke Math. J. 22 (1955), 75-94.

[3] O. Demanze, On subnormality and formal subnormality for tuples of unbounded operators, Integral Equations Operator Theory 46 (2003), 267-284.

[4] -, A subnormality criterion for unbounded tuples of operators, Acta Sci. Math. (Szeged) 69 (2003), 773-787.

[5] N. Dunford and J. T. Schwartz, Linear Operators, Part II, Interscience, New York, 1963.

[6] M. R. Embry, A generalization of the Halmos-Bram criterion for subnormality, Acta Sci. Math. (Szeged) 35 (1973), 61-64.

[7] P. Găvruţă and N. Suciu, A characterization of subnormal pairs, in: Operator Theory, Operator Algebra and Related Topics (Timişoara, 1996), Theta, Bucureşti, 1997, 149-157.

[8] P. R. Halmos, Normal dilations and extensions of operators, Sum. Brasil. Math. 2 (1950), 125-134.

[9] T. Itô, On the commutative family of subnormal operators, J. Fac. Sci. Hokkaido Univ. 14 (1958), 1-15. 
[10] Z. J. Jabłoński and J. Stochel, Subnormality and operator multidimensional moment problems, J. London Math. Soc. 71 (2005), 438-466.

[11] A. Lubin, Weighted shifts and commuting normal extensions, J. Austral. Math. Soc. Ser. A 27 (1979), 17-26.

[12] M. Putinar and F.-H. Vasilescu, Solving moment problems by dimensional extension, Ann. of Math. 149 (1999), 1087-1107.

[13] W. Rudin, Analyse fonctionnelle, 2ème éd., Ediscience international, Paris, 1965.

[14] J. Stochel and F. H. Szafraniec, On normal extensions of unbounded operators I, J. Operator Theory 14 (1985), 31-55.

[15] - - - On normal extensions of unbounded operators II, Acta Sci. Math. (Szeged) 53 (1989), 153-177.

[16] —, - , The complex moment problem and subnormality: A polar decomposition approach, J. Funct. Anal. 159 (1998), 432-491.

[17] F. H. Szafraniec, Subnormality in the quantum harmonic oscillator, Comm. Math. Phys. 210 (2000), 323-334.

[18] T. T. Trent, New conditions for subnormality, Pacific J. Math. 93 (1981), 459-464.

[19] F.-H. Vasilescu, Moment problems for multi-sequences of operators, J. Math. Anal. Appl. 219 (1998), 246-259.

[20] - Operator moment problems in unbounded sets, in: Recent Advances in Operator Theory and Related Topics (Szeged, 1999), Oper. Theory Adv. Appl. 127, Birkhäuser, 2001, 613-638.

[21] - Extensions of unbounded symmetric multioperators, in: Operator Theory and Banach Algebras (Rabat, 1999), Theta, Bucureşti, 2003.

Laboratoire de Mathématiques, UMR 8524

Université des sciences et technologies de Lille

59655 Villeneuve d'Ascq Cedex, France

E-mail: Olivier.Demanze@math.univ-lille1.fr

Received May 28, 2004

Revised version June 7, 2005 